\title{
A Home-Network Service System Based on User's Situation Information in Ubiquitous Environment ${ }^{\star}$
}

\author{
Yongyun Cho, Joohyun Han, Jaeyoung Choi, and Chae-Woo Yoo \\ School of Computing, Soongsil University, \\ 1-1 Sangdo-dong, Dongjak-gu, Seoul 156-743, Korea \\ \{yycho, jhhan\}@ss.ssu.ac.kr, \\ $\{$ choi, cwyoo\}@comp.ssu.ac.kr
}

\begin{abstract}
In this paper, we propose a home-network service system that can support home services appropriate to user's situation information in ubiquitous computing environments. The suggested system uses a uWDL workflow service scenario describing a user's situation information as service execution constraints to support context-aware home services. The suggested system consists of a context handler and a context mapper. The context handler represents contexts described in a uWDL document as a context subtree, which expresses not only context data but also relation information among services into the fields of its node. The context mapper uses a context comparison algorithm for context comparison between context subtrees and user's situation information. The algorithm can distinguish contexts that have the same values but different types with user's contexts and selects a context that has all together values and types entirely equal to those of user's contexts.
\end{abstract}

\section{Introduction}

For a smart home, execution of all the home services must be dependent on user's situation contexts, which are dynamically generated in ubiquitous environments [1. uWDL (ubiquitous Workflow Definition Language) is a workflow language based on a structural context model which expresses context information as transition constraints of workflow services 2]. Through a uWDL workflow service scenario, an user can describe what services must be executed according to situation information. For execution of context-aware services, we need a method that can recognize contexts in a scenario and select a service correspondent with situation information.

In this paper, we present a uWDL-based home-network system that represents contexts described in a uWDL workflow service scenario document as rule-based context subtrees, and derives service transition according to user's state information in ubiquitous environments.

\footnotetext{
* This work was supported by Korea Research Foundation Grant (KRF-2004-005D00172).
} 


\section{Related Work}

Context in a ubiquitous environment means any information that can be used to characterize the situation of an entity [3. In ubiquitous environments, context can be expressed with a RDF-based triplet form [2]. RDF(Resource Description Framework) 44 is a language to describe resource's meta-data and it expresses a resource as a triplet of subject, predicate, objective $\}$. The existing workflow languages, such as BPEL4WS [5], WSFL [6], and XLANG [7], do not include any element to describe context information in ubiquitous computing environments as transition conditions of services.

uWDL 2] can describe context information as transition conditions of services through the <context $>$ element consisting of the knowledge-based triplet - subject, verb, and object. The uWDL reflects the advantages of current workflow languages such as BPEL4WS, WSFL, and XLANG, and also contains rule-based expressions to interface with the DAML+OIL ontology language [8].

\section{A uWDL-Based Home-Network Service System}

\subsection{A System Architecture}

Figure 1 shows the architecture of the suggested uWDL scenario-based smarthome system, which is aware of user's situation information in ubiquitous computing environments.

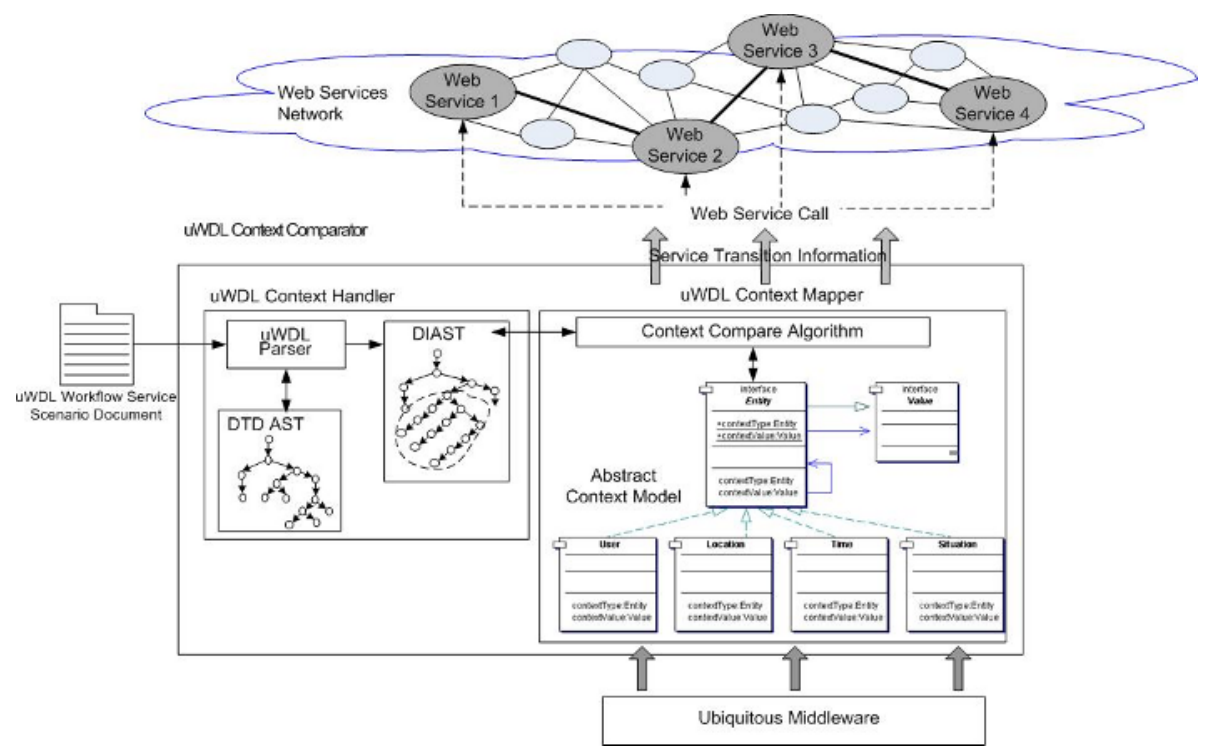

Fig. 1. A smart-home system's architecture based in a uWDL workflow service scenario 
As shown in Figure 1, the suggested system supports context-aware home services using a uWDL service scenario, in which an user's situation information is described as a services execution condition. A uWDL context mapper in Figure 1 uses a context subtree for context comparison with user's situation information. Through the context comparison, the uWDL context mapper searches home service networks for a home service appropriate to a user's situation information generated from ubiquitous computing environments. If it finds a service corresponding to a specific user's situation information, the uWDL context mapper calls the service to offer context-aware home service to users.

\subsection{A uWDL Context Handler and a uWDL Context Mapper}

As a result of a parsing, the uWDL handler makes a DIAST (Document Instance Abstract Syntax Tree) that represents the structure information of a $\mathrm{uWDL}$ document as a tree data structure. At this time, a context described as RDFbased triplet entity in a uWDL scenario is constructed as a subtree of the parse tree. Contexts that the context mapper uses for the comparison are described in a triplet based on RDF. Context information from the sensor network can be embodied as a triplet consisting of subject, verb and object according to the structural context model based in RDF. The context mapper extracts context types and values of the entity objectified from sensors. It then compares the context types and values of the objectified entity with those of the DIAST's subtree elements related to the entity. In the comparison, if the context types and values in the entity coincide with the counterpart in the DIAST's subtree, the context mapper drives the service workflow. For that, we define a context embodied with a structural context model from the sensor network as OC and a context described in a uWDL scenario as UC. OC means a context objectified with the structural context model, and UC means a context described in a uWDL scenario. Also, OCS and UCS that mean each set of OC and UC.

\section{Experiments and Results}

For an experiment, we have developed an uWDL scenario for home-network services through PDA. The example scenario is as follows: John has a plan to go back his home at 8:00 PM, take a warm bath, and then watch a recorded TV program, which he wants to see after a bath. When John arrives to his apartment, an RFID sensor above the apartment door transmits John's basic context information (such as name, notebook's IP address) to the uWDL home server. If the conditions, such as user location, situation, and current time, are satisfied with contexts described in the uWDL workflow service scenario, then the server will prepare warm water. When he sits the sofa in the living room after he finishes a bath, the service engine will turn on the power of TV in the living room and play the TV program that was recorded already. For the experiment, we use a Pentium IV 3.0 GHZ computer with 1GB memory based in Windows XP OS as a uWDL home service engine and a PDA with 512MB memory based in Windows CE for the experiment. Figure 2 shows the result. 

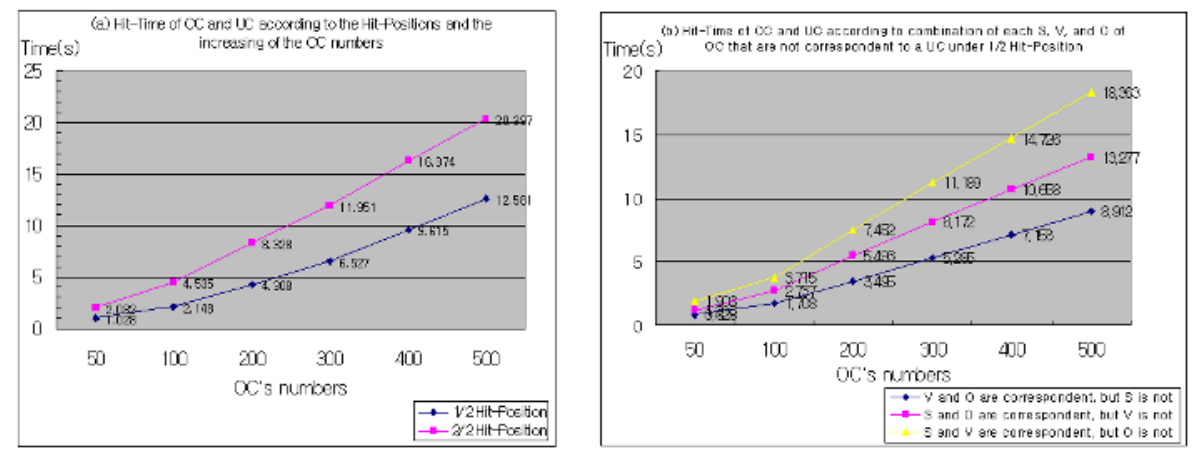

Fig. 2. A result of hit-time of $\mathrm{OC}$ and UC according to hit-position and conditions of OC's $s, v$, and $o$

\section{Conclusion}

In this paper, we presented a home-network service system that can recognize a uWDL workflow service scenario document, and can drive home services according to a user's situation information. Through experiments, we defined contexts described in a uWDL scenario as OC and contexts objectified from ubiquitous computing environments. We showed an experiment in which the uWDL mapper compared contexts of UCSs and OCSs through the context comparison algorithm, and measured hit-times and service transition accuracy to verify the efficiency of the algorithm. Through the results, we found that the hit-times were reasonable in spite of increment in the OCs amounts. Therefore, this uWDL context comparator will contribute greatly to the development of the context-aware application programs in ubiquitous computing environments.

\section{References}

1. W. Keith Edwards and Rebecca E. Grinter, Ubicomp 2001, LNCS 2201, pp. 256-272, 2001.

2. Joohyun Han, Yongyun Cho, Jaeyoung Choi: Context-Aware Workflow Language based on Web Services for Ubiquitous Computing, ICCSA 2005, LNCS 3481, pp. 1008-1017, (2005)

3. Anind k. Dey: Understanding and Using Context, Personal and Ubiquitous Computing, Vol 5, Issue 1, pp.69-78 (2001)

4. W3C: RDF/XML Syntax Specification, W3C Recommendation (2004)

5. Tony Andrews, Francisco Curbera, Yaron Goland: Business Process Execution Language for Web Services, BEA Systems, Microsoft Corp., IBM Corp., Version 1.1 (2003)

6. Frank Leymann: Web Services Flow Language (WSFL 1.0). IBM (2001)

7. Satish Thatte: XLANG Web Services for Business Process Design, Microsoft Corp. (2001)

8. R. Scott Cost, Tim Finin: ITtalks: A Case Study in the Semantic Web and DAML+OIL, University of Maryland, Baltimore County, IEEE (2002) 1094-7167 